\title{
A Kalman rank condition for the localized distributed controllability of a class of linear parabolic systems.
}

\author{
Ammar-Khodja Farid, Benabdallah Assia \\ Dupaix Cédric ${ }^{\ddagger}$ González-Burgos Manuel ${ }^{\S}$
}

January 26, 2009

\begin{abstract}
We present a generalization of the Kalman rank condition to the case of $n \times n$ linear parabolic systems with constant coefficients and diagonalizable diffusion matrix. To reach the result, we are led to prove a global Carleman estimate for the solutions of a scalar $2 n$-order parabolic equation and deduce from it an observability inequality for our adjoint system.
\end{abstract}

AMS 2000 subject classification: 93B05, 93B07, 35K05, 35K55, 35R30.

Keywords: Kalman condition, control, observability, Carleman estimates, reaction diffusion systems.

\section{Introduction. The main result}

Controllability of linear ordinary differential systems is well-known. In particular we have at our disposal the famous Kalman rank condition (see for example $\left[17\right.$, Chapter 2, p. 35]), that is to say, if $n, m \in \mathbb{N}$ with $n, m \geq 1$ and $A \in \mathcal{L}\left(\mathbb{R}^{n}\right)$ and $B \in \mathcal{L}\left(\mathbb{R}^{m}, \mathbb{R}^{n}\right)$, then the linear ordinary differential system $Y^{\prime}=A Y+B u$ is controllable at time $T>0$ if and only if

$$
\operatorname{rank}[A \mid B]=\operatorname{rank}\left[A^{n-1} B\left|A^{n-2} B\right| \cdots \mid B\right]=n .
$$

The main goal of our work is to give an extension of this algebraic condition to a class of parabolic systems of partial differential equations where the controls

\footnotetext{
*Laboratoire de Mathématiques UMR 6623, Université de Franche-Comté, 16, route de Gray, 25030 Besançon cedex, France. E-mail: fammarkh@univ-fcomte.fr.

${ }^{\dagger}$ CMI-LATP, UMR 6632, Université de Provence, Technopôle Château-Gombert, 39, rue F. Joliot-Curie, 13453 Marseille cedex 13, France. E-mail: assia@cmi.univ-mrs.fr

¥Laboratoire de Mathématiques UMR 6623, Université de Franche-Comté, 16, route de Gray, 25030 Besançon cedex, France. E-mail: cedric.dupaix@univ-fcomte.fr.

$\S$ Dpto, E.D.A.N., Universidad de Sevilla, Aptdo. 1160, 41080 Sevilla, Spain. E-mail: manoloburgos@us.es. Supported by D.G.E.S. (Spain), grant MTM2006-07932.
} 
act on a subdomain of the domain where the parabolic system is posed. To this end, let $\Omega \subset \mathbb{R}^{d}$ be a bounded open set with a $C^{2}$-boundary $\partial \Omega$, let $\omega \subset \Omega$ be a nonempty open subset and assume $T>0$. Let us consider the second order elliptic self adjoint operator given by

$$
R=\sum_{i, j=1}^{N} \partial_{i}\left(r_{i j}(x) \partial_{j}\right)+c(x)
$$

with

$$
\left\{\begin{array}{l}
r_{i j} \in W^{1, \infty}(\Omega), \quad c \in L^{\infty}(\Omega) \quad(1 \leq i, j \leq d), \\
r_{i j}(x)=r_{j i}(x) \quad \text { a.e. in } \Omega,
\end{array}\right.
$$

and the coefficients $r_{i j}$ satisfy the uniform elliptic condition

$$
\sum_{i, j=1}^{d} r_{i j}(x) \xi_{i} \xi_{j} \geq a_{0}|\xi|^{2}, \quad \forall \xi \in \mathbb{R}^{d}, \quad \text { a.e. in } \Omega
$$

for a positive constant $a_{0}$.

For $n, m \in \mathbb{N}^{*}$ and $T>0$, we consider the following $n \times n$ parabolic system

$$
\left\{\begin{array}{l}
\partial_{t} y=(D R+A) y+B v 1_{\omega} \text { in } \Omega_{T}=\Omega \times(0, T), \\
y=0 \text { on } \Sigma_{T}=\partial \Omega \times(0, T), \quad y(\cdot, 0)=y_{0}(\cdot) \text { in } \Omega,
\end{array}\right.
$$

where $D \in \mathcal{L}\left(\mathbb{R}^{n}\right), A=\left(a_{i j}\right)_{1 \leq i, j \leq n} \in \mathcal{L}\left(\mathbb{R}^{n}\right)$ and $B \in \mathcal{L}\left(\mathbb{R}^{m}, \mathbb{R}^{n}\right)$ are given, $v \in L^{2}\left(\Omega_{T}\right)^{m}\left(\Omega_{T}=\Omega \times(0, T)\right)$ and $y_{0}=\left(y_{0, i}\right)_{1 \leq i \leq n} \in L^{2}(\Omega)^{n}$. In (1.4), $y=\left(y_{i}\right)_{1 \leq i \leq n}$ is the state variable while $1_{\omega}$ denotes the characteristic function of the open subset $\omega$.

All along this work we will assume that the diffusion matrix $D$ is diagonalizable with positive real eigenvalues, i.e., for $J=\operatorname{diag}\left(d_{i}\right)_{n \times n}$ with $d_{1}, d_{2}, \ldots, d_{n}>$ 0 , one has

$$
D=P^{-1} J P, \text { with } P \in \mathcal{L}\left(\mathbb{R}^{n}\right), \operatorname{det} P \neq 0
$$

Let us observe that, thanks to the assumptions on the operator $R$ and the diffusion matrix $D$, for every $y_{0} \in L^{2}(\Omega)^{n}$ and $v \in L^{2}\left(\Omega_{T}\right)$ system (1.4) possesses a unique solution $y \in L^{2}\left(0, T ; H_{0}^{1}(\Omega)^{n}\right) \cap C^{0}\left([0, T] ; L^{2}(\Omega)^{n}\right)$.

The exact controllability to zero of system (1.4) reads as follows: For given $T>0$ and $y_{0} \in L^{2}(\Omega)^{n}$ can we find $v \in L^{2}\left(\Omega_{T}\right)^{m}$ such that the corresponding solution $y$ of (1.4) satisfies $y(T)=0$ a.e. in $\Omega$ ? In the linear case, this property is equivalent to the exact controllability to the trajectories, that is to say, to the following property: for every trajectory $y^{*} \in C^{0}\left([0, T] ; L^{2}(\Omega)^{n}\right)$ of $(1.4)$ (i.e., a solution to (1.4) corresponding to $v^{*} \in L^{2}\left(\Omega_{T}\right)^{m}$ and $\left.y_{0}^{*} \in L^{2}(\Omega)^{n}\right)$ and $y_{0} \in L^{2}(\Omega)^{n}$, there exists a control $v \in L^{2}\left(\Omega_{T}\right)^{m}$ such that the corresponding solution $y$ to (1.4) satisfies

$$
y(\cdot, T)=y^{*}(\cdot, T) \quad \text { in } \quad \Omega .
$$


Finally, it will be said that (1.4) is approximately controllable in $L^{2}(\Omega)^{n}$ at time $T$ if, for any $y_{0}, y_{d} \in L^{2}(\Omega)^{n}$ and any $\varepsilon>0$, there exists a control function $v \in L^{2}\left(\Omega_{T}\right)^{m}$ such that the solution $y \in C^{0}\left([0, T] ; L^{2}(\Omega)^{n}\right)$ to (1.4) satisfies

$$
\left\|y(\cdot, T)-y_{d}\right\|_{L^{2}(\Omega)^{n}} \leq \varepsilon .
$$

Let us remark that in the scalar case, i.e., when $n=1$, the controllability properties of system (1.4) are well known (see for instance [18], [11] and [10]). To be precise, under the previous assumptions and for every $\omega \subset \Omega$ and $T>0$, if $B \neq 0$, system (1.4) is exactly controllable to the trajectories, null controllable and approximately controllable in $L^{2}(\Omega)$ at time $T>0$. Therefore we will concentrate on the study of the controllability properties of system (1.4) in the non scalar case and all along this paper we will assume that $n \geq 2$ and $m \geq 1$.

The study of the controllability properties of system (1.4) is a mathematical interesting problem. Moreover, systems as (1.4) arise, for example, in the modeling of biological phenomena as the growth of tumors. In this context, the control $v$ represents a therapy which only acts on some of the variables of the systems.

There are few results providing sufficient conditions for the exact controllability to the trajectories of system (1.4) (when $n>1$ and $1 \leq m<n$ ) and most of them are proved for $n=2$ and $B=(1,0)^{*}$ : see, for instance, [22], [1], [4], [12] and [14] (also see [9] and [7] for similar controllability results of cascade system of parabolic-hyperbolic equations). The most general result in this direction seems to be the one in [13] where the authors study a cascade parabolic system of $n$ equations. Finally we also point out [3] where the authors provide a sufficient condition on the coupling matrices for obtaining the null controllability on the time interval $\left(T_{0}, T_{1}\right)$, with $0 \leq T_{0}<T_{1} \leq T$, of the non-autonomous parabolic linear system:

$$
\left\{\begin{array}{l}
\partial_{t} y=L(t) y+A(t) y+B(t) v 1_{\omega} \text { in } \Omega \times\left(T_{0}, T_{1}\right) \\
y=0 \text { on } \partial \Omega \times\left(T_{0}, T_{1}\right)
\end{array}\right.
$$

where $A(\cdot) \in C^{n-1}\left([0, T] ; \mathcal{L}\left(\mathbb{R}^{n}\right)\right), B(\cdot) \in C^{n}\left([0, T] ; \mathcal{L}\left(\mathbb{R}^{m}, \mathbb{R}^{n}\right)\right)$ and $L(t)$ is a scalar time-dependent elliptic second order operator given by

$$
\left\{\begin{aligned}
L(t) y(x, t) & =-\sum_{i, j=1}^{N} \frac{\partial}{\partial x_{i}}\left(a_{i j}(x, t) \frac{\partial y}{\partial x_{j}}(x, t)\right)+\sum_{i=1}^{N} b_{i}(x, t) \frac{\partial y}{\partial x_{i}}(x, t) \\
& +d(x, t) y(x, t),
\end{aligned}\right.
$$

where $a_{i j} \in W^{1, \infty}\left(\Omega_{T}\right), b_{i}, d \in L^{\infty}\left(\Omega_{T}\right), a_{i j}(x, t)=a_{j i}(x, t)$ a.e. in $\Omega_{T}(1 \leq$ $i, j \leq N)$, and

$$
\sum_{i, j=1}^{N} a_{i j}(x, t) \xi_{i} \xi_{j} \geq \widetilde{a}_{0}|\xi|^{2}, \quad \forall \xi \in \mathbb{R}^{N}, \quad \text { a.e. in } \Omega_{T},
$$

for a positive constant $\widetilde{a}_{0}$. 
For obtaining a necessary and sufficient condition for the controllability of system (1.4), let us introduce the Kalman operator. We denote by $L$ the operator given by $L:=D R+A$. Thanks to (1.5), we deduce $D(L)=D(R)^{n}=$ $\left(H^{2}(\Omega) \cap H_{0}^{1}(\Omega)\right)^{n}$. Then the Kalman operator associated with $(L, B)$ is the matrix operator

$$
\left\{\begin{array}{l}
\mathcal{K}:=[L \mid B]: D(\mathcal{K}) \subset L^{2}(\Omega)^{n m} \rightarrow L^{2}(\Omega)^{n}, \text { with } \\
D(\mathcal{K}):=\left\{u \in L^{2}(\Omega)^{n m}: \mathcal{K} u \in L^{2}(\Omega)^{n}\right\},
\end{array}\right.
$$

where

$$
[L \mid B]=\left[L^{n-1} B\left|L^{n-2} B\right| \ldots|L B| B\right] .
$$

Our main result is the following:

Theorem 1.1. Let us assume that D satisfies (1.5). Then, system (1.4) (with $R$ given by (1.2)) is exactly controllable to trajectories at any time $T$ if and only if the Kalman operator $\mathcal{K}$ satisfies

$$
\operatorname{Ker}\left(\mathcal{K}^{*}\right)=\{0\} .
$$

As said above the Kalman rank condition (1.1) is a necessary and sufficient condition for the controllability of linear ordinary differential systems (see [24]). The first attempt to extend this condition to the case of an infinite dimensional systems are described and discussed in [23].

The closest result to Theorem 1.1 we know is due to H. Leiva [19] which gives a necessary and sufficient condition for the approximate controllability of (1.4) in the case in which $D$ is a diagonalizable matrix whose eigenvalues have positive real part, $A \equiv 0$ and $B(x) \in L^{2}\left(\Omega ; \mathcal{L}\left(\mathbb{R}^{m} ; \mathbb{R}^{n}\right)\right)$.

Thanks to (1.2), it is well known that the operator $-R$ with Dirichlet boundary conditions admits a sequence of eigenvalues

$$
0<\lambda_{1}<\lambda_{2} \leq \ldots \leq \lambda_{p} \leq \lambda_{p+1} \leq \ldots, \quad \lambda_{p} \rightarrow \infty,
$$

such that the associated sequence of normalized eigenfunctions $\left(\phi_{p}\right)_{p \in \mathbb{N}^{*}}$ is an orthonormal basis of $L^{2}(\Omega)$. With the previous notations, we will see that, in fact, condition (1.6) can be easily checked since it is equivalent to the algebraic condition

$$
\operatorname{rank}\left[\left(-\lambda_{p} D+A\right) \mid B\right]=n, \quad \forall p \geq 1
$$

(see Proposition 2.2; we have used the notation

$$
\left.\left[\left(-\lambda_{p} D+A\right) \mid B\right]=\left[\left(-\lambda_{p} D+A\right)^{n-1} B\left|\left(-\lambda_{p} D+A\right)^{n-2} B\right| \cdots \mid B\right]\right) .
$$

Remark 1.1. It is interesting to observe that system (1.4) can be exactly controlled to the trajectories with one control force even if $A \equiv 0$. Indeed, let us assume that $D=\operatorname{diag}\left(d_{i}\right)_{n \times n}$, with $d_{i}>0$ for every $1 \leq i \leq n$, and 
$B=\left(b_{1}, \ldots, b_{n}\right)^{*} \in \mathbb{R}^{n}$. Then, in order to apply Theorem 1.1 we should check condition (1.6), i.e., condition (1.7). So,

$$
\left[\left(-\lambda_{p} D+A\right) \mid B\right]=\left[\begin{array}{cccc}
\left(-\lambda_{p} d_{1}\right)^{n-1} b_{1} & \left(-\lambda_{p} d_{1}\right)^{n-2} b_{1} & \cdots & b_{1} \\
\left(-\lambda_{p} d_{2}\right)^{n-1} b_{2} & \left(-\lambda_{p} d_{2}\right)^{n-2} b_{2} & \cdots & b_{2} \\
\vdots & \vdots & \ddots & \vdots \\
\left(-\lambda_{p} d_{n}\right)^{n-1} b_{n} & \left(-\lambda_{p} d_{n}\right)^{n-2} b_{n} & \cdots & b_{n}
\end{array}\right] \in \mathcal{L}\left(\mathbb{R}^{n}\right)
$$

and (1.7) holds if and only if $b_{i} \neq 0$ for every $i$ and the diffusion coefficients $d_{i}$ are distinct.

Remark 1.2. Let us remark that the controllability properties of system (1.4) when the diffusion matrix $D$ given by $D=\beta_{0} I d$, with $\beta_{0}>0$, have been already studied in [3]. To be precise, in [3] it is proved that system (1.4) (with $\left.D=\beta_{0} I d\right)$ is exactly controllable to the trajectories if and only if $\operatorname{rank}[A \mid B]=n$. On the other hand, for $D=\beta_{0} I d$, it is not difficult to see that condition (1.7) is independent of the eigenvalues $\lambda_{p}$ and is equivalent to $\operatorname{rank}[A \mid B]=n$. As a consequence, in this work we recover the results on controllability of system (1.4) proven in [3] in the case $D=\beta_{0} I d$, with $\beta_{0}>0, A \in \mathcal{L}\left(\mathbb{R}^{n}\right)$ and $B \in \mathcal{L}\left(\mathbb{R}^{m} ; \mathbb{R}^{n}\right)$. It is also interesting to point out that the techniques developed in this work are completely different from those used in [3].

In order to study the exact controllability to the trajectories of system (1.4), we will consider the corresponding adjoint problem

$$
\left\{\begin{array}{l}
-\partial_{t} \varphi=\left(D^{*} R+A^{*}\right) \varphi \text { in } \Omega_{T} \\
\varphi=0 \text { on } \Sigma_{T}, \quad \varphi(x, T)=\varphi_{0} \text { in } \Omega
\end{array}\right.
$$

where $\varphi_{0} \in L^{2}(\Omega)^{n}$. It is by now well known that the exact controllability to the trajectories of system (1.4) is equivalent to the existence of a positive constant $C$ such that, for every $\varphi_{0} \in L^{2}(\Omega)^{n}$, the solution $\varphi \in C^{0}\left([0, T] ; L^{2}(\Omega)^{n}\right)$ to the adjoint system (1.8) satisfies the observability inequality:

$$
\|\varphi(\cdot, 0)\|_{L^{2}(\Omega)^{n}}^{2} \leq C \iint_{\omega_{T}}\left|B^{*} \varphi(x, t)\right|^{2},
$$

where we have introduced the notation $\omega_{T}=\omega \times(0, T)$.

The observability inequality (1.9) will be deduced from an appropriate global Carleman inequality satisfied by the solutions to the adjoint problem (1.8) (see Theorem 1.3). In order to state this Carleman inequality we will reason as follows: firstly we will show a global Carleman inequality (see Theorem 1.2) which bounds a weighted global integral of $\mathcal{K}^{*} \varphi$ by means of a weighted local integral of $B^{*} \varphi$ ( $\varphi$ is the solution to the adjoint problem (1.8) corresponding to $\left.\varphi_{0}\right)$. Secondly, and using condition (1.6), we will be able to deduce from the previous inequality the appropriate global Carleman inequality for the solutions to the adjoint problem and, in particular, (1.9).

Our second result reads as follows: 
Theorem 1.2. Let us assume that $D$ satisfies (1.5). Then, given $\tau \in \mathbb{R}$ and $k \geq(n-1)(2 n-1)$, there exist a positive function $\beta_{0} \in C^{2}(\bar{\Omega})$ (only depending on $\Omega$ and $\omega$ ), $r=r(n) \in \mathbb{N}$ and two positive constants $C$ and $\sigma$ (only depending on $\Omega, \omega, n,\left(r_{i j}\right)_{1 \leq i, j \leq N}, D, A, k$ and $\left.\tau\right)$ such that for every $\varphi_{0} \in L^{2}(\Omega)^{n}$ the corresponding solution $\varphi$ to (1.8) satisfies

$$
\int_{0}^{T}(s \rho)^{\tau} e^{\frac{-2 s M_{0}}{t(T-t)}}\left\|R^{k} \mathcal{K}^{*} \varphi\right\|_{L^{2}(\Omega)^{n m}}^{2} \leq C \iint_{\omega_{T}}(s \rho)^{\tau+K+r} e^{-2 s \eta}\left|B^{*} \varphi\right|^{2},
$$

for every $s \geq \sigma\left(T+T^{2}+T^{2}\|c\|_{\infty}^{2 / 3}\right)$. In (1.10), $\rho, \eta, M_{0}$ and $K$ are respectively given by: $\rho(t)=t^{-1}(T-t)^{-1}, \eta(x, t)=\beta_{0}(x) / t(T-t), M_{0}=\max _{\bar{\Omega}} \beta_{0}$ and $K=4 k+n-4$.

Remark 1.3. Let us observe that if $\varphi_{0} \in L^{2}(\Omega)^{n}$, the corresponding solution $\varphi$ to (1.8) satisfies $\varphi \in C^{\infty}\left((0, T] ; D\left(R^{k}\right)^{n}\right)$, for every $k \geq 1$. Indeed, $A$ is a constant matrix and the operator $D^{*} R+A^{*}$ with domain $D\left(D^{*} R\right)=$ $\left(H^{2}(\Omega) \cap H_{0}^{1}(\Omega)\right)^{n}$, is the generator of an analytic semigroup on $L^{2}(\Omega)^{n}$ (see Section 2 and Subsection 3.2.

Finally, in our third result we state a global Carleman inequality for the solutions to (1.8). At this point we use condition (1.6) in an essential way:

Theorem 1.3. In addition to the assumptions in Theorem 1.2, we assume condition (1.6). Then, given $\tau \in \mathbb{R}$ and $k \geq(n-1)(2 n-1)$, there exist two positive constants $C$ and $\sigma$ (only depending on $\Omega, \omega, n,\left(r_{i j}\right)_{1 \leq i, j \leq N}, D, A, k$ and $\tau$ ) such that for every $\varphi_{0} \in L^{2}(\Omega)^{n}$ the corresponding solution $\varphi$ to (1.8) satisfies

$$
\iint_{\Omega_{T}}(s \rho)^{\tau} e^{\frac{-2 s M_{0}}{t(T-t)}}\left|R^{k-(n-1)(2 n-1)} \varphi\right|^{2} \leq C \iint_{\omega_{T}}(s \rho)^{\tau+K+r} e^{-2 s \eta}\left|B^{*} \varphi\right|^{2},
$$

for every $s \geq \sigma\left(T+T^{2}+T^{2}\|c\|_{\infty}^{2 / 3}\right)$. In (1.11), $\rho, \eta, M_{0}, K$ and $r(n)$ are as in Theorem 1.2.

The plan of the paper is the following: In section 2, we will address some properties of the unbounded Kalman operator $\mathcal{K}$ and prove the continuity (in a suitable sense) of its inverse, when it exists. Then, in section 3, a proof of Theorem 1.2 is given using a global Carleman estimate for a scalar parabolic equation of order $2 n$ (see Theorem 3.2). In section 4 we give some comments and open problems.

The results that we present in this work have been announced in [2].

\section{Properties of the Kalman operator}

Define

$$
\mathcal{D}=\bigcap_{p=0}^{\infty} D\left(R^{p}\right)
$$


It is well-known that $\mathcal{D}$ is dense in $D\left(R^{p}\right)$ for every $p \geq 0$ and straightforward that $\mathcal{D}^{n m} \subset D(\mathcal{K})$. Thus, $\overline{D(\mathcal{K})}=L^{2}(\Omega)^{n m}$ and $\mathcal{K}^{*}$ is well-defined from $D\left(\mathcal{K}^{*}\right) \subset L^{2}(\Omega)^{n}$ into $L^{2}(\Omega)^{n m}$. The formal adjoint operator of $\mathcal{K}$, again denoted by $\mathcal{K}^{*}$, is given by

$$
\mathcal{K}^{*}=\left[\begin{array}{c}
B^{*}\left(L^{*}\right)^{n-1} \\
\vdots \\
B^{*} L^{*} \\
B^{*}
\end{array}\right]
$$

and it coincides with the adjoint operator of $\mathcal{K}$ on $\mathcal{D}^{n}$.

This section will be devoted to the proof of the following result which contains two crucial properties of the Kalman operator $\mathcal{K}$ :

Theorem 2.1. There exists a positive constant $C$ such that:

1. Continuity of $\mathcal{K}$ and $\mathcal{K}^{*}:$ If $u \in D\left(R^{n-1}\right)^{n m} \quad\left(\right.$ resp. $\left.u \in D\left(R^{n-1}\right)^{n}\right)$ then $\mathcal{K} u \in L^{2}(\Omega)^{n}$ (resp. $\left.\mathcal{K}^{*} u \in L^{2}(\Omega)^{n m}\right)$ and

$$
\|\mathcal{K} u\|_{L^{2}(\Omega)^{n}}^{2} \leq C\left\|R^{n-1} u\right\|_{L^{2}(\Omega)^{n m}}^{2},
$$

$\left(\right.$ resp. $\left.\left\|\mathcal{K}^{*} u\right\|_{L^{2}(\Omega)^{n m}}^{2} \leq C\left\|R^{n-1} u\right\|_{L^{2}(\Omega)^{n}}^{2}\right)$.

2. Invertibility of $\mathcal{K}^{*}$ and continuity of the inverse: Assume that condition (1.6) is fulfilled and let $k \in \mathbb{N}$ with $k \geq(2 n-1)(n-1)$. Then, for every $\varphi \in L^{2}(\Omega)^{n}$ satisfying $\mathcal{K}^{*} \varphi \in D\left(R^{k}\right)^{n m}$, one has $\varphi \in$ $D\left(R^{k-(2 n-1)(n-1)}\right)^{n}$ and

$$
\left\|R^{k-(2 n-1)(n-1)} \varphi\right\|_{L^{2}(\Omega)^{n}}^{2} \leq C\left\|R^{k} \mathcal{K}^{*} \varphi\right\|_{L^{2}(\Omega)^{n m}}^{2} .
$$

Remark 2.1. Let us remark that, in general, the operator $\mathcal{K}$ (resp. $\mathcal{K}^{*}$ ) is not onto, even if $\operatorname{Ker} \mathcal{K}=\{0\}$. Indeed, assume that $n \geq 2, m=1$ and take, for instance, $D=\operatorname{diag}\left(d_{i}\right)_{n \times n}\left(d_{1}, d_{2}, \ldots, d_{n}>0\right)$ and $B \equiv e_{1}$. We consider $f \equiv\left(A e_{1}\right) g \in L^{2}(\Omega)^{n}$ with $g \in H_{0}^{1}(\Omega)$ such that $R g \notin L^{2}(\Omega)$. If we pose the equation

$$
x \in D(\mathcal{K}) \text { such that } \mathcal{K} x=f,
$$

we readily see that it admits the solution $x=\left(0, \ldots, 0, x_{1}, x_{0}\right)^{t}$ with $x_{1}=g \in$ $L^{2}(\Omega)$ and $x_{0}=-d_{1} R x_{1} \notin L^{2}(\Omega)$ :

$$
\mathcal{K} x=L B x_{1}+B x_{0} \equiv\left(d_{1} R x_{1}+x_{0}\right) e_{1}+\left(A e_{1}\right) x_{1} \equiv f .
$$

Thus, the previous equation has no solution in $D(\mathcal{K})$ and $\mathcal{K}$ is not onto.

Before proving Theorem 2.1, let us introduce some notations. We recall that $\left(\lambda_{p}\right)_{p \in \mathbb{N}^{*}}$ is the sequence of eigenvalues of the operator $-R$ with Dirichlet 
boundary conditions and $\left(\phi_{p}\right)_{p \in \mathbb{N}^{*}}$ the associated sequence of eigenfunctions. For any $j, p \in \mathbb{N}^{*}$, we consider the projection operator

$$
P_{p}^{j}: \Psi=\left(\Psi_{k}\right)_{1 \leq k \leq j} \in L^{2}(\Omega)^{j} \rightarrow P_{p}^{j}(\Psi)=\left(\left(\Psi_{k}, \phi_{p}\right)\right)_{1 \leq k \leq j} \in \mathbb{R}^{j},
$$

where $(\cdot, \cdot)$ stands for the scalar product in $L^{2}(\Omega)$. All along this paper we denote by $|\cdot|$ the euclidian norm in $\mathbb{R}^{j}$. Thus, if $j \in \mathbb{N}^{*}$, a characterization of $\mathcal{D}^{j}$ is:

$$
\mathcal{D}^{j}=\left\{\Psi=\sum_{p \geq 1} \Psi_{p} \phi_{p}: \Psi_{p} \in \mathbb{R}^{j} \text { and } \sum_{p \geq 1} \lambda_{p}^{2 m}\left|\Psi_{p}\right|^{2}<\infty, \forall m \geq 0\right\} .
$$

For $p \in \mathbb{N}^{*}$, let us denote $L_{p}=-\lambda_{p} D+A \in \mathcal{L}\left(\mathbb{R}^{n}\right)$ and

$$
\mathcal{K}_{p}=\left[L_{p} \mid B\right]=\left[L_{p}^{n-1} B\left|L_{p}^{n-2} B\right| \cdots \mid B\right] \in \mathcal{L}\left(\mathbb{R}^{n m}, \mathbb{R}^{n}\right) .
$$

It is not difficult to show the equalities

$$
\left\{\begin{array}{l}
L\left(b \phi_{p}\right)=\left(L_{p} b\right) \phi_{p}, \quad \forall b \in \mathbb{R}^{n}, \quad \forall p \geq 1 \text { and } \\
\mathcal{K}\left(b \phi_{p}\right)=\left(\mathcal{K}_{p} b\right) \phi_{p}, \quad \forall b \in \mathbb{R}^{n m}, \quad \forall p \geq 1
\end{array}\right.
$$

From these identities and taking into account that $L$ and $\mathcal{K}$ are closed unbounded operators, a direct computation gives

$$
\left\{\begin{array}{l}
L y=\sum_{p \geq 1} L_{p} P_{p}^{n}(y) \phi_{p}, \quad \forall y \in D(L), \\
\mathcal{K} u=\sum_{p \geq 1} \mathcal{K}_{p} P_{p}^{n m}(u) \phi_{p}, \quad \forall u \in D(\mathcal{K}),
\end{array}\right.
$$

and then,

$$
D(\mathcal{K})=\left\{u \in L^{2}(\Omega)^{n m}: \sum_{p \geq 1}\left|\mathcal{K}_{p} P_{p}^{n m}(u)\right|^{2}<\infty\right\} .
$$

In the same way, we also have:

$$
\left\{\begin{array}{l}
\mathcal{K}^{*}=\sum_{p \geq 1} \mathcal{K}_{p}^{*} P_{p}^{n}(\cdot) \phi_{p}, \quad \mathcal{K}_{p}^{*}=\left[L_{p} \mid B\right]^{*} \\
D\left(\mathcal{K}^{*}\right)=\left\{\varphi \in L^{2}(\Omega)^{n}: \sum_{p \geq 1}\left|\mathcal{K}_{p}^{*} P_{p}^{n}(\varphi)\right|^{2}<\infty\right\} .
\end{array}\right.
$$

Finally, we define the operator $\mathcal{K} \mathcal{K}^{*}: D\left(\mathcal{K} \mathcal{K}^{*}\right) \subset L^{2}(\Omega)^{n} \rightarrow L^{2}(\Omega)^{n}$, with domain

$$
D\left(\mathcal{K} \mathcal{K}^{*}\right)=\left\{\varphi \in L^{2}(\Omega)^{n}: \mathcal{K}^{*} \varphi \in D(\mathcal{K}), \quad \mathcal{K} \mathcal{K}^{*} \varphi \in L^{2}(\Omega)^{n}\right\}
$$


Note that $\mathcal{K} \mathcal{K}^{*}$ is a closed unbounded operator and, again, a simple calculation provides the equalities:

$$
\left\{\begin{array}{l}
\mathcal{K K}^{*} \varphi=\sum_{p \geq 1} \mathcal{K}_{p} \mathcal{K}_{p}^{*} P_{p}^{n}(\varphi) \phi_{p} \\
D\left(\mathcal{K} \mathcal{K}^{*}\right)=\left\{\varphi \in L^{2}(\Omega)^{n}: \sum_{p \geq 1}\left|\mathcal{K}_{p}^{*} P_{p}^{n}(\varphi)\right|^{2}, \sum_{p \geq 1}\left|\mathcal{K}_{p} \mathcal{K}_{p}^{*} P_{p}^{n}(\varphi)\right|^{2}<\infty\right\}
\end{array}\right.
$$

With these notations, it is easy to show that:

Proposition 2.2. The following conditions are equivalent:
1. $\operatorname{Ker}\left(\mathcal{K}^{*}\right)=\{0\}$.
2. $\operatorname{Ker}\left(\mathcal{K K}^{*}\right)=\{0\}$.
3. $\operatorname{det} \mathcal{K}_{p} \mathcal{K}_{p}^{*} \neq 0$ for every $p \geq 1$.

We are now ready to prove Theorem 2.1.

\section{Proof of Theorem 2.1.}

1. From the expression of $\mathcal{K}_{p}\left(\right.$ resp. $\left.\mathcal{K}_{p}^{*}\right)$, it is not difficult to prove that for some positive constant we have

$$
\left|\mathcal{K}_{p} b\right| \leq C \lambda_{p}^{n-1}|b|, \quad \forall b \in \mathbb{R}^{n m} \text { and } p \geq 1,
$$

(resp. $\left|\mathcal{K}_{p}^{*} b\right| \leq C \lambda_{p}^{n-1}|b|$, for every $b \in \mathbb{R}^{n}$ and $p \geq 1$ ). Now, if $u \in D\left(R^{n-1}\right)^{n m}$, one has

$$
\left\{\begin{aligned}
\|\mathcal{K} u\|_{L^{2}(\Omega)^{n}}^{2} & =\sum_{p \geq 1}\left|\mathcal{K}_{p} P_{p}^{n m}(u)\right|^{2} \leq C \sum_{p \geq 1} \lambda_{p}^{2(n-1)}\left|P_{p}^{n m}(u)\right|^{2} \\
& =C\left\|R^{n-1} u\right\|_{L^{2}(\Omega)^{n m}}^{2} .
\end{aligned}\right.
$$

The proof for $\mathcal{K}^{*}$ can be obtained in a similar way.

2. Let us consider $\varphi \in L^{2}(\Omega)^{n}$ with $\mathcal{K}^{*} \varphi \in D\left(R^{r}\right)^{n m}$ and let us set $u \equiv \mathcal{K}^{*} \varphi \in$ $D\left(R^{r}\right)^{n m}$. This last equality can be equivalently written as

$$
\mathcal{K}_{p}^{*} P_{p}^{n}(\varphi)=P_{p}^{n m}(u), \quad \forall p \geq 1
$$

Applying $\mathcal{K}_{p}$, we get:

$$
\mathcal{K}_{p} \mathcal{K}_{p}^{*} P_{p}^{n}(\varphi)=\mathcal{K}_{p} P_{p}^{n m}(u), \quad \forall p \geq 1
$$

Let us remark that the elements of the matrix $[-\lambda D+A \mid B][-\lambda D+A \mid B]^{*}$ are polynomials of degree $2(n-1)$. Therefore, we can write

$$
[-\lambda D+A \mid B][-\lambda D+A \mid B]^{*}=\left[M_{1}(\lambda), \cdots, M_{n}(\lambda)\right],
$$


with $M_{j}(\lambda)=\left(p_{i j}(\lambda)\right)_{1 \leq i \leq n}, 1 \leq j \leq n$, and $p_{i j}(\lambda)$ polynomials of degree $2(n-1)$. For $\lambda \in \mathbb{R}, Z \in \mathbb{R}^{n}$ and $1 \leq i \leq n$ we introduce

$$
\left\{\begin{array}{l}
F(\lambda)=\operatorname{det}[-\lambda D+A \mid B][-\lambda D+A \mid B]^{*} \text { and } \\
F_{i}(\lambda, Z)=\operatorname{det}\left[M_{1}(\lambda), \cdots, M_{i-1}(\lambda), Z, M_{i+1}(\lambda), \cdots, M_{n}(\lambda)\right] .
\end{array}\right.
$$

The function $F(\lambda)$ is a polynomial of degree $2 n(n-1)$ and taking into account the condition (1.6) and Proposition 2.2 we deduce

$$
F\left(\lambda_{p}\right)=\operatorname{det} \mathcal{K}_{p} \mathcal{K}_{p}^{*} \neq 0, \quad \forall p \geq 1 .
$$

Thus, $F(\lambda)$ is not the null polynomial and we deduce that, in particular, there is $C>0$ such that

$$
\left|F\left(\lambda_{p}\right)\right| \geq C>0, \quad \forall p \geq 1 .
$$

On the other hand, $F_{i}(\lambda, Z)$ is a linear functional with respect to $Z$ and a polynomial of degree $2(n-1)^{2}$ with respect to $\lambda$. We can easily verify that, for some $C>0$, we have

$$
\left|F_{i}(\lambda, Z)\right| \leq C(|\lambda|+1)^{2(n-1)^{2}}|Z|, \quad \forall(\lambda, Z) \in \mathbb{R} \times \mathbb{R}^{n} .
$$

Coming back to (2.14) we have (Kramer's rule)

$$
\left(P_{p}^{n}(\varphi)\right)_{i}=\frac{F_{i}\left(\lambda_{p}, \mathcal{K}_{p} P_{p}^{n m}(u)\right)}{F\left(\lambda_{p}\right)}, \quad 1 \leq i \leq n .
$$

This last inequality together with (2.13) and the properties of $F$ and $F_{i}$ give

$$
\left|P_{p}^{n}(\varphi)\right| \leq C \lambda_{p}^{2(n-1)^{2}}\left|\mathcal{K}_{p} P_{p}^{n m}(u)\right| \leq C \lambda_{p}^{(2 n-1)(n-1)}\left|P_{p}^{n m}(u)\right|, \quad \forall p \geq 1 .
$$

Since $u \equiv \mathcal{K}^{*} \varphi \in D\left(R^{r}\right)^{n m}$ and $k \geq(2 n-1)(n-1)$ we deduce

$$
\sum_{p \geq 1} \lambda^{2 k-2(2 n-1)(n-1)}\left|P_{p}^{n}(\varphi)\right|^{2} \leq \sum_{p \geq 1} \lambda_{p}^{2 k}\left|P_{p}^{n m}(u)\right|^{2}<\infty,
$$

i.e. (2.12). This ends the proof of Theorem 2.1.

The previous proof in particular shows the following property.

Corollary 2.3. Either there exists $p_{0} \in \mathbb{N}^{*}$ such that $\operatorname{rank} \mathcal{K}_{p}=n$ for every $p>p_{0}$ or $\operatorname{rank} \mathcal{K}_{p}<n$ for every $p \in \mathbb{N}^{*}$.

Proof. The alternative comes from the fact that either the polynomial $F(\lambda)$ is identically 0 , either it is far from 0 for any $\lambda$ sufficiently large.

Remark 2.2. From the previous corollary we deduce that if for some $\widehat{p} \geq 1$ the condition $\operatorname{rank}\left[\left(-\lambda_{\widehat{p}} D+A\right) \mid B\right]=n$ holds, then there exists $p_{0} \geq 1$ such that

$$
\operatorname{rank}\left[\left(-\lambda_{p} D+A\right) \mid B\right]=n, \quad \forall p \geq p_{0} .
$$

Under the previous hypothesis we will see in the last section that we can show that system (1.4) is exactly controllable to the trajectories when condition (1.6) is not fulfilled and the initial data $y_{0}$ and $y_{0}^{*}$ belong to an appropriate infinitedimensional subspace of $L^{2}(\Omega)^{n}$. 


\section{Proof of Theorems 1.2 and 1.3. The Carleman inequality}

This section is devoted to prove Theorems 1.2 and 1.3. To this end, we will also prove a Carleman inequality for the solutions of a scalar parabolic equation of order $2 n$. We will deduce Theorem 1.2 from this result and Theorem 1.3 combining this result and condition (1.6).

First of all let us recall the notations for Carleman's estimate (see [11] and [16]). We introduce the following functions:

$$
\left\{\begin{array}{rlrl}
\eta(x, t) & :=\frac{\beta_{0}(x)}{t(T-t)}, & & \forall(x, t) \in \Omega_{T}=\Omega \times(0, T), \\
\rho(t) & :=\frac{1}{t(T-t)}, & \forall t \in(0, T) .
\end{array}\right.
$$

where $\beta_{0} \in C^{2}(\bar{\Omega})$. Then, for $\tau \in \mathbb{R}$ we define the functional

$$
I(\tau, \varphi)=\iint_{\Omega_{T}}(s \rho)^{\tau-1} e^{-2 s \eta}\left(\left|\varphi_{t}\right|^{2}+|R \varphi|^{2}+(s \rho)^{2}|\nabla \varphi|^{2}+(s \rho)^{4}|\varphi|^{2}\right) .
$$

Let $\omega^{\prime}$ be a non-empty open subset of $\Omega$ such that $\omega^{\prime} \subset \subset \omega \subset \Omega$. Using the notation $\omega_{T}^{\prime}=\omega^{\prime} \times(0, T)$, we have the following

Theorem 3.1. Let $\tau \in \mathbb{R}$ and $d>0$ be fixed. Then, there exist a positive function $\beta_{0} \in C^{2}(\bar{\Omega})$ (only depending on $\Omega$ and $\omega^{\prime}$ ) and two positive constants $\widetilde{\sigma}_{0}>0$ and $\widetilde{C}_{0}$ (which only depend on $\Omega, \omega^{\prime},\left(r_{i j}\right)_{1<i, j<N}, d$ and $\tau$ ) such that for every $\varphi \in L^{2}\left(0, T ; H_{0}^{1}(\Omega)\right)$ with $\partial_{t} \varphi \pm d R \varphi \in L^{2}\left(\Omega_{T}\right)$, the following (Carleman) estimate holds

$$
I(\tau, \varphi) \leq \widetilde{C}_{0}\left(\iint_{\Omega_{T}}(s \rho)^{\tau} e^{-2 s \eta}\left|\partial_{t} \varphi \pm d R \varphi\right|^{2}+\iint_{\omega_{T}^{\prime}}(s \rho)^{\tau+3} e^{-2 s \eta}|\varphi|^{2}\right)
$$

for all $s \geq \widetilde{s}_{0}=\widetilde{\sigma}_{0}\left(T+T^{2}+T^{2}\|c\|_{\infty}^{2 / 3}\right)$.

The proof of this result can be found in [16] although the authors do not specify the way the constant $s_{0}$ depends on $T$. This explicit dependence can be obtained arguing as in [10].

\subsection{A Carleman estimate for a scalar parabolic equation of order $2 n$}

In this subsection we will consider $\phi$, with $R^{i} \partial_{t}^{j} \phi \in L^{2}\left(0, T ; H^{2}(\Omega) \cap H_{0}^{1}(\Omega)\right)$ for every $i, j \in \mathbb{N}$, a solution of the following scalar parabolic equation of order $2 n$

$$
\left\{\begin{array}{l}
P\left(\partial_{t}, \nabla\right) \phi=0 \text { in } \Omega_{T} \\
R^{k} \phi=0 \text { on } \Sigma_{T}, \quad \forall k \geq 0
\end{array}\right.
$$


where $P\left(\partial_{t}, \nabla\right)$ is the operator given by $P\left(\partial_{t}, \nabla\right)=\operatorname{det}\left(\partial_{t} I d+D^{*} R+A^{*}\right)$. Using (1.5) we can compute this determinant and obtain

$$
\left\{\begin{aligned}
P\left(\partial_{t}, \nabla\right) & =\operatorname{det}\left(\partial_{t} I d+P^{*} J\left(P^{*}\right)^{-1} R+A^{*}\right)=\operatorname{det}\left(\partial_{t} I d+J R+\left(P^{*}\right)^{-1} A P^{*}\right) \\
& =P_{n} \cdots P_{1}+\sum_{p=2}^{n-1} \sum_{1 \leq i_{1}<\cdots<i_{p} \leq n} \alpha_{i_{1}, \ldots, i_{p}} P_{i_{1}} \ldots P_{i_{p}}+\sum_{i=1}^{n} \alpha_{i} P_{i}+\alpha, \\
P_{i} & \equiv \partial_{t}+d_{i} R, \quad 1 \leq i \leq n, \quad d_{i}>0,
\end{aligned}\right.
$$

and $\alpha_{i_{1}, \ldots, i_{p}}, \alpha_{i}, \alpha \in \mathbb{R}$ depend on the entries of $D$ and $A$.

The objective of this section is to prove a Carleman estimate for the function $\phi$, solution to (3.16):

Theorem 3.2. Let us fix $k_{1}, k_{2} \in \mathbb{N}$ and $\tau_{0} \in \mathbb{R}$. Then, there exist two positive constants $C_{0}$ and $\sigma_{0}$ (only depending on $\Omega, \omega, n,\left(r_{i j}\right)_{1 \leq i, j \leq N}, D, A, \tau_{0}, k_{1}$ and $k_{2}$ ) and $r=r(n) \in \mathbb{N}$ such that the following inequality

$$
\sum_{i=0}^{k_{1}} \sum_{j=0}^{k_{2}} \Im\left(\tau_{0}-4(i+j), R^{i} \partial_{t}^{j} \phi\right) \leq C_{0} \iint_{\omega_{T}}(s \rho)^{\tau_{0}+r} e^{-2 s \eta}|\phi|^{2},
$$

holds for all $s \geq \sigma_{0}\left(T+T^{2}+T^{2}\|c\|_{\infty}^{2 / 3}\right)$ and for every $\phi$ solution to (3.16) that satisfies $R^{i} \partial_{t}^{j} \phi \in L^{2}\left(0, T ; H^{2}(\Omega) \cap H_{0}^{1}(\Omega)\right)$ for every $i, j \in \mathbb{N}$. In (3.17), $\Im(\tau, \phi)$ is given by

$$
\left\{\begin{aligned}
\Im(\tau, \phi) & =I(\tau+3(n-1), \phi)+\sum_{i=1}^{n} I\left(\tau+3(n-2), P_{i} \phi\right) \\
& +\sum_{p=2}^{n-1} \sum_{1 \leq i_{1}<\cdots<i_{p} \leq n} I\left(\tau+3(n-p-1), P_{i_{p}} \ldots P_{i_{1}} \phi\right),
\end{aligned}\right.
$$

where $I(\cdot, \cdot)$ is defined in (3.15).

Proof: All along the proof, $C$ will be a generic constants that may depend on $\Omega$, $\omega, n$, the matrix $\left(r_{i j}\right)_{1 \leq i, j \leq N}, D, A, \tau_{0}, k_{1}$ and $k_{2}$. For the reader's convenience we will divide the proof in several steps:

Step 1: Let us denote

$$
F(\phi)=-\left(\sum_{p=2}^{n-1} \sum_{1 \leq i_{1}<\cdots<i_{p} \leq n} \alpha_{i_{1}, \ldots, i_{p}} P_{i_{1}} \ldots P_{i_{p}}+\sum_{i=1}^{n} \alpha_{i} P_{i}+\alpha\right) \phi
$$

and consider the following change of variables:

$$
\left\{\begin{array}{l}
\psi_{1}=\phi \\
\psi_{i}=P_{i-1} \psi_{i-1}=\left(\partial_{t}+d_{i-1} R\right) \psi_{i-1}, \quad 2 \leq i \leq n .
\end{array}\right.
$$


Taking into account the regularity assumptions on $\phi,(3.16)$ and (3.18), it is not difficult to check that $\psi_{i}, F(\phi) \in L^{2}\left(\Omega_{T}\right)$ for every $i, 1 \leq i \leq n$, and $\Psi=\left(\psi_{1}, \ldots, \psi_{n}\right)^{*}$ satisfies the cascade system

$$
\left\{\begin{array}{c}
\left(\partial_{t}+d_{1} R\right) \psi_{1}=\psi_{2} \text { in } \Omega_{T}, \\
\left(\partial_{t}+d_{2} R\right) \psi_{2}=\psi_{3} \text { in } \Omega_{T}, \\
\vdots \\
\left(\partial_{t}+d_{n} R\right) \psi_{n}=F(\phi) \text { in } \Omega_{T}, \\
\psi_{i}=0 \text { on } \Sigma_{T}, \quad \forall i: 1 \leq i \leq n .
\end{array}\right.
$$

In a first step, we will establish the inequality

$$
\left\{\begin{aligned}
\sum_{i=1}^{n} I\left(\tau_{0}+3(n-i), \psi_{i}\right) & \leq C\left(\sum_{i=1}^{n} \iint_{\omega_{T}^{\prime}}(s \rho)^{\tau_{0}+3(n-i+1)} e^{-2 s \eta}\left|\psi_{i}\right|^{2}\right. \\
& \left.+\iint_{\Omega_{T}}(s \rho)^{\tau_{0}} e^{-2 s \eta}|F(\phi)|^{2}\right)
\end{aligned}\right.
$$

for every $s \geq \sigma_{1}\left(T+T^{2}+T^{2}\|c\|_{\infty}^{2 / 3}\right)$ and where $C$ and $\sigma_{1}$ are positive constants which depend $\Omega, \omega, n,\left(r_{i j}\right)_{1 \leq i, j \leq N}, D$ and $\tau_{0}$.

Indeed, applying Theorem 3.1 to each function $\psi_{i}(1 \leq i \leq n)$, solution to system (3.20), with $\tau=\tau_{0}+3(n-i)$, we obtain the existence of a constant $\sigma_{1}>0$ (depending on $\Omega, \omega, n,\left(r_{i j}\right)_{1 \leq i, j \leq N}, D$ and $\tau_{0}$ ) such that

$$
\left\{\begin{aligned}
I\left(\tau_{0}+3(n-i), \psi_{i}\right) & \leq C\left(\iint_{\Omega_{T}}(s \rho)^{\tau_{0}+3(n-i)} e^{-2 s \eta}\left|\psi_{i+1}\right|^{2}\right. \\
& \left.+\iint_{\omega_{T}^{\prime}}(s \rho)^{\tau_{0}+3(n-i+1)} e^{-2 s \eta}\left|\psi_{i}\right|^{2}\right), \quad 1 \leq i \leq n-1,
\end{aligned}\right.
$$

and

$$
I\left(\tau_{0}, \psi_{n}\right) \leq C\left(\iint_{\Omega_{T}}(s \rho)^{\tau_{0}} e^{-2 s \eta}|F(\phi)|^{2}+\iint_{\omega_{T}^{\prime}}(s \rho)^{\tau_{0}+3} e^{-2 s \eta}\left|\psi_{n}\right|^{2}\right),
$$

hold for every $s \geq \sigma_{1}\left(T+T^{2}+T^{2}\|c\|_{\infty}^{2 / 3}\right)$. Thus a suitable combination of these inequalities leads to (3.21).

Note that for the moment, $F(\phi)$ depends itself on $\phi=\psi_{1}$. So in the next step, we will prove that we can get rid of the local terms and the term corresponding to $F(\phi)$ in the right-hand-side of (3.21).

Step 2: In this step we will reduce the number of observations (local integrals) in inequality (3.21). To be precise, we will prove that there exist $C$ and $\sigma_{2}\left(\sigma_{2}\right.$ 
depending on $\Omega, \omega, n,\left(r_{i j}\right)_{1 \leq i, j \leq N}, D, A$ and $\left.\tau_{0}\right)$ such that

$$
\left\{\begin{aligned}
I\left(\tau_{0}+3(n-1), \phi\right)+\sum_{i=1}^{n} I\left(\tau+3(n-2), P_{i} \phi\right) \\
+\sum_{p=2}^{n-1} \sum_{1 \leq i_{1}<\cdots<i_{p} \leq n} I\left(\tau+3(n-p-1), P_{i_{p}} \ldots P_{i_{1}} \phi\right) \\
\leq C \iint_{\omega_{T}}(s \rho)^{\tau_{0}+r} e^{-2 s \eta}|\phi|^{2}
\end{aligned}\right.
$$

for every $s \geq \sigma_{2}\left(T+T^{2}+T^{2}\|c\|_{\infty}^{2 / 3}\right)$.

The starting point of the proof is estimate (3.21). For $i=1, \ldots, n$ we introduce a family of bounded open sets $\left(\omega^{i}\right)_{1 \leq i \leq n}$ and an associated family of truncation functions $\left(\delta_{i}\right)_{1 \leq i \leq n}$ satisfying

$$
\omega^{n}:=\omega^{\prime} \subset \subset \omega^{n-1} \subset \subset \cdots \subset \subset \omega^{1}:=\omega \subset \Omega,
$$

$\delta_{i} \in C_{c}^{2}\left(\omega^{i-1}\right), \delta_{i}=1$ in $\bar{\omega}^{i}$ and $0 \leq \delta_{i} \leq 1$ in $\omega^{i-1}$.

Let us fix $\ell \geq 3$ and $k \in\{2, \ldots, n\}$. Let us also consider the equation for $\psi_{k-1}$ in system (3.20), i.e., the equation $\partial_{t} \psi_{k-1}+d_{k-1} R \psi_{k-1}=\psi_{k}$. Multiplying then this equation by $\theta \delta_{k} \psi_{k}$ with $\theta(x, t)=(s \rho(x, t))^{\tau_{0}+\ell} e^{-2 s \eta}$, we obtain that

$$
\left\{\begin{aligned}
\iint_{\omega_{T}^{k}}(s \rho)^{\tau_{0}+\ell} e^{-2 s \eta}\left|\psi_{k}\right|^{2} & \leq \iint_{\Omega_{T}} \theta \delta_{k}\left|\psi_{k}\right|^{2} \\
= & \iint_{\Omega_{T}} \theta \delta_{k}\left(\partial_{t} \psi_{k-1}+d_{k-1} R \psi_{k-1}\right) \psi_{k}=I_{1}+I_{2} .
\end{aligned}\right.
$$

In order to bound $I_{1}$ and $I_{2}$, we will reason as in [22] and [12]. All along this second step we will assume that $s \geq \sigma_{1}\left(T+T^{2}+T^{2}\|c\|_{\infty}^{2 / 3}\right)$. In particular $s \geq \sigma_{1}\left(T+T^{2}\right)$ and, then, for every $\mu, \nu \in \mathbb{R}, \nu \leq \mu$, and for every $(x, t) \in \Omega_{T}$, one has

$$
\left\{\begin{array}{l}
(s \rho)^{\nu} \leq(s \rho)^{\mu}, \quad\left|\nabla\left[(s \rho)^{\nu} e^{-2 s \eta}\right]\right| \leq C(s \rho)^{\nu+1} e^{-2 s \eta} \\
\left|\partial_{t}\left[(s \rho)^{\nu} e^{-2 s \eta}\right]\right|+\sum_{i, j=1}^{N}\left|\partial_{i j}^{2}\left[(s \rho)^{\nu} e^{-2 s \eta}\right]\right| \leq C(s \rho)^{\nu+2} e^{-2 s \eta}
\end{array}\right.
$$

Thus

$$
\left\{\begin{aligned}
I_{1} & =-\iint_{\Omega_{T}} \delta_{k}\left(\partial_{t} \theta\right) \psi_{k-1} \psi_{k}-\iint_{\Omega_{T}} \delta_{k} \theta \psi_{k-1} \partial_{t} \psi_{k} \\
& \leq C\left(\iint_{\Omega_{T}} \delta_{k}(s \rho)^{\tau_{0}+\ell+2} e^{-2 s \eta}\left|\psi_{k-1}\right|\left|\psi_{k}\right|+\iint_{\Omega_{T}} \delta_{k} \theta\left|\psi_{k-1}\right|\left|\partial_{t} \psi_{k}\right|\right. \\
& \leq \frac{\varepsilon}{2} I\left(\tau_{0}+3(n-k), \psi_{k}\right)+\frac{C}{\varepsilon} \iint_{\omega_{T}^{k-1}}(s \rho)^{\tau_{0}+2 \ell-3(n-k)+1} e^{-2 s \eta}\left|\psi_{k-1}\right|^{2},
\end{aligned}\right.
$$


where $\varepsilon>0$ is fixed and where $\omega_{T}^{k-1}=\omega^{k-1} \times(0, T)$. On the other hand,

$$
I_{2}=d_{k-1} \iint_{\Omega_{T}} \psi_{k-1} R\left(\delta_{k} \theta \psi_{k}\right) \text {. }
$$

But using (3.24),

$$
\left\{\begin{aligned}
\left|R\left(\delta_{k} \theta \psi_{k}\right)\right| & =\mid\left(R \delta_{k}\right) \theta \psi_{k}+2 \sum_{i, j=1}^{N} r_{i j}\left(\partial_{j} \delta_{k}\right)\left(\partial_{i} \theta\right) \psi_{k}+\delta_{k}(R \theta) \psi_{k} \\
& +2 \sum_{i, j=1}^{N} r_{i j}\left(\partial_{i} \delta_{k}\right) \theta\left(\partial_{j} \psi_{k}\right)+\delta_{k} \theta\left(R \psi_{k}\right) \\
& +2 \sum_{i, j=1}^{N} r_{i j} \delta_{k}\left(\partial_{i} \theta\right)\left(\partial_{j} \psi_{k}\right) \mid \\
& \leq C 1_{\omega_{T}^{k-1}}(s \rho)^{\tau_{0}+\ell} e^{-2 s \eta}\left[(s \rho)^{2}\left|\psi_{k}\right|+s \rho\left|\nabla \psi_{k}\right|+\left|R \psi_{k}\right|\right],
\end{aligned}\right.
$$

and therefore

$$
I_{2} \leq \frac{\varepsilon}{2} I\left(\tau_{0}+3(n-k)\right)+\frac{C}{\varepsilon} \iint_{\omega_{T}^{k-1}}(s \rho)^{\tau_{0}+2 \ell-3(n-k)+1} e^{-2 s \eta}\left|\psi_{k-1}\right|^{2} .
$$

Coming back to (3.23) we get

$$
\iint_{\omega_{T}^{k}}(s \rho)^{\tau_{0}+\ell} e^{-2 s \eta}\left|\psi_{k}\right|^{2} \leq \varepsilon I\left(\tau_{0}+3(n-k)\right)+\frac{C}{\varepsilon} \iint_{\omega_{T}^{k-1}}(s \rho)^{K} e^{-2 s \eta}\left|\psi_{k-1}\right|^{2}
$$

with $\varepsilon>0$ and $K=\tau_{0}+2 \ell-3(n-k)+1$.

In order to show (3.22) we apply this last inequality with $\ell=3, k=n$ and $\varepsilon=1 / 2 C(C$ as in (3.21)). So, from (3.21), we infer

$$
\left\{\begin{aligned}
\sum_{i=1}^{n} I\left(\tau_{0}+3(n-i), \psi_{i}\right) & \leq C\left(\sum_{i=1}^{n-1} \iint_{\omega_{T}^{n-1}}(s \rho)^{\tau_{0}+K(i)} e^{-2 s \eta}\left|\psi_{i}\right|^{2}\right. \\
& \left.+\iint_{\Omega_{T}}(s \rho)^{\tau_{0}} e^{-2 s \eta}|F(\phi)|^{2}\right)
\end{aligned}\right.
$$

for every $s \geq \sigma_{1}\left(T+T^{2}+T^{2}\|c\|_{\infty}^{2 / 3}\right)$. In this inequality, $C$ is a new positive constant and $K(i)=\max \{7,3(n-i+1)\}(1 \leq i \leq n-1)$.

We can repeat this process and show the existence of a constant $C>0$ and an integer $r=r(n) \in \mathbb{N}$ such that

$$
\left\{\begin{aligned}
\sum_{i=1}^{n} I\left(\tau_{0}+3(n-i), \psi_{i}\right) & \leq C\left(\iint_{\omega_{T}}(s \rho)^{\tau_{0}+r} e^{-2 s \eta}\left|\psi_{1}\right|^{2}\right. \\
& \left.+\iint_{\Omega_{T}}(s \rho)^{\tau_{0}} e^{-2 s \eta}|F(\phi)|^{2}\right),
\end{aligned}\right.
$$


for all $s \geq \sigma_{1}\left(T+T^{2}+T^{2}\|c\|_{\infty}^{2 / 3}\right)$, which in view of (3.19) implies

$$
\left\{\begin{array}{l}
I\left(\tau_{0}+3(n-1), \phi\right)+\sum_{i=2}^{n} I\left(\tau_{0}+3(n-i), P_{i-1} \ldots P_{1} \phi\right) \\
\leq C\left(\iint_{\omega_{T}}(s \rho)^{\tau_{0}+r} e^{-2 s \eta}|\phi|^{2}+\iint_{\Omega_{T}}(s \rho)^{\tau_{0}} e^{-2 s \eta}|F(\phi)|^{2}\right) .
\end{array}\right.
$$

Note, at this level, that we have got rid of the local terms in the right-handside of (3.21) but the left-hand-side of (3.25) does not contains enough terms to absorb the term corresponding to $F(\phi)$.

So let $\Pi$ denote then any permutation of $\{1,2, \ldots, n\}$ and consider, instead of (3.19), the new change of variable

$$
\left\{\begin{array}{l}
\psi_{1}=\phi \\
\psi_{i}=P_{\Pi(i-1)} \psi_{i-1}=\left(\partial_{t}+d_{\Pi(i-1)} R\right) \psi_{i-1}, \quad 2 \leq i \leq n .
\end{array}\right.
$$

Then system (3.20) becomes

$$
\left\{\begin{aligned}
\left(\partial_{t}+d_{\Pi(1)} R\right) \psi_{1} & =\psi_{2} \text { in } \Omega_{T} \\
\left(\partial_{t}+d_{\Pi(2)} R\right) \psi_{2} & =\psi_{3} \text { in } \Omega_{T} \\
& \vdots \\
\left(\partial_{t}+d_{\Pi(n)} \Delta\right) \psi_{n} & =F(\phi) \text { in } \Omega_{T} \\
\psi_{i}=0 \text { on } \Sigma_{T} &
\end{aligned}\right.
$$

and the same procedure as above leads to a similar estimate as (3.25) which reads then

$$
\left\{\begin{array}{l}
I\left(\tau_{0}+3(n-1), \phi\right)+\sum_{i=2}^{n} I\left(\tau_{0}+3(n-i), P_{\Pi(i-1)} \ldots P_{\Pi(1)} \phi\right) \\
\leq C\left(\iint_{\omega_{T}}(s \rho)^{\tau_{0}+r} e^{-2 s \eta}|\phi|^{2}+\iint_{\Omega_{T}}(s \rho)^{\tau_{0}} e^{-2 s \eta}|F(\phi)|^{2}\right),
\end{array}\right.
$$

for all $s \geq \sigma_{1}\left(T+T^{2}+T^{2}\|c\|_{\infty}^{2 / 3}\right)$, where $C$ is a new positive constant.

Thus, considering all such possible permutations with associated change of variable, we finally obtain

$$
\left\{\begin{aligned}
I\left(\tau_{0}+3(n-1), \phi\right)+ & \sum_{i=1}^{n} I\left(\tau_{0}+3(n-2), P_{i} \phi\right) \\
& +\sum_{p=2}^{n-1} \sum_{1 \leq i_{1}<\cdots<i_{p} \leq n} I\left(\tau_{0}+3(n-p-1), P_{i_{p}} \ldots P_{i_{1}} \phi\right) \\
& \leq C\left(\iint_{\omega_{T}}(s \rho)^{\tau_{0}+r} e^{-2 s \eta}|\phi|^{2}+\iint_{\Omega_{T}}(s \rho)^{\tau_{0}} e^{-2 \eta}|F(\phi)|^{2}\right),
\end{aligned}\right.
$$


where $s \geq \sigma_{1}\left(T+T^{2}+T^{2}\|c\|_{\infty}^{2 / 3}\right)$ and $C$ is a new positive constant.

Now, we are going to prove that the term corresponding to $F(\phi)$ can be absorbed by the left-hand-side of (3.26). Using the definition of $F(\phi)$ (see (3.18)) we have that:

$$
\left\{\begin{aligned}
\iint_{\Omega_{T}}(s \rho)^{\tau_{0}} e^{-2 s \eta}|F(\phi)|^{2} & \leq C \iint_{\Omega_{T}}(s \rho)^{\tau_{0}} e^{-2 s \eta}\left(|\phi|^{2}+\sum_{i=1}^{n}\left|P_{i} \phi\right|^{2}\right. \\
& \left.+\sum_{p=2}^{n-1} \sum_{1 \leq i_{1}<\cdots<i_{p} \leq n}\left|P_{i_{1}} \ldots P_{i_{p}} \phi\right|^{2}\right)
\end{aligned}\right.
$$

where $C$ is a new positive constant (observe that $C$ also depends on $A$ through the coefficients $\left.\alpha_{i_{1}, \ldots, i_{p}}, \alpha_{i}, \alpha\right)$.

Finally, choosing $s \geq \sigma_{2}\left(T+T^{2}+T^{2}\|c\|_{\infty}^{2 / 3}\right)$, with $\sigma_{2}$ depending on $\Omega, \omega$, $n,\left(r_{i j}\right)_{1 \leq i, j \leq N}, D, A$ and $\tau_{0}$, we deduce

$$
C(s \rho)^{\tau_{0}} \leq \frac{1}{2}(s \rho)^{\tau_{0}+3(n-p)}, \quad \forall p: 0 \leq p \leq n-1,
$$

and (3.22) from (3.26) and (3.27).

Remark 3.1. In view of steps 1 and 2 , instead of (3.16), one can consider the following system

$$
\left\{\begin{array}{l}
P\left(\partial_{t}, \nabla\right) \phi=f \text { in } \Omega_{T}, \\
R^{k} \phi=0 \text { on } \Sigma_{T}, \quad \forall k \geq 0,
\end{array}\right.
$$

with $f \in L^{2}\left(\Omega_{T}\right)$. It is a direct application to deduce:

$$
\left\{\begin{aligned}
I\left(\tau_{0}+3(n-1), \phi\right)+\sum_{i=1}^{n} I\left(\tau+3(n-2), P_{i} \phi\right) \\
+\sum_{p=2}^{n-1} \sum_{1 \leq i_{1}<\cdots<i_{p} \leq n} I\left(\tau+3(n-p-1), P_{i_{p}} \ldots P_{i_{1}} \phi\right) \\
\leq C \iint_{\omega_{T}}(s \rho)^{\tau_{0}+r} e^{-2 s \eta}|\phi|^{2}+\iint_{\Omega_{T}}(s \rho)^{\tau_{0}} e^{-2 s \eta}|f|^{2}
\end{aligned}\right.
$$

for every $s \geq \sigma_{2}\left(T+T^{2}+T^{2}\|c\|_{\infty}^{2 / 3}\right)$.

Step 3: It is interesting to remark that, thanks to the regularity assumptions imposed on $\phi$, if $1 \leq i \leq k_{1}$ and $1 \leq j \leq k_{2}, R^{i} \partial_{t}^{j} \phi$ also fulfills equation (3.16) and the two previous steps developed for $\phi$ can be repeated for $R^{i} \partial_{t}^{j} \phi$. Therefore, from (3.22), if we take $\tau \in \mathbb{R}$ we deduce the existence of two positive constants $C_{\tau}$ and $\sigma_{\tau}$, which depend on $\Omega, \omega, n,\left(r_{i j}\right)_{1 \leq i, j \leq N}, D, A$ and $\tau$, such that

$$
\Im\left(\tau, R^{i} \partial_{t}^{j} \phi\right) \leq C_{\tau} \iint_{\omega_{T}}(s \rho)^{\tau+r} e^{-2 s \eta}\left|R^{i} \partial_{t}^{j} \phi\right|^{2},
$$


for every $s \geq \sigma_{\tau}\left(T+T^{2}+T^{2}\|c\|_{\infty}^{2 / 3}\right)$ (we recall that $\Im(\tau, \phi)$ is defined in the statement of Theorem 3.2).

On the other hand, it is easy to check that for any $\psi \in L^{2}(0, T ; D(R))$ with $\partial_{t} \psi \in L^{2}\left(\Omega_{T}\right)$

$$
\iint_{\omega_{T}}(s \rho)^{\tau+r} e^{-2 s \eta}\left(|R \psi|^{2}+\left|\partial_{t} \psi\right|^{2}\right) \leq I(\tau+4, \psi) \leq \mathfrak{I}(\tau+4, \psi),
$$

$(I(\cdot, \cdot)$ is given by $(3.15))$. Thus, if $s \geq \sigma_{\tau}\left(T+T^{2}+T^{2}\|c\|_{\infty}^{2 / 3}\right)$, one has

$$
\left\{\begin{array}{l}
\Im\left(\tau, R^{i} \partial_{t}^{j} \phi\right) \leq C_{\tau} \Im\left(\tau+4, R^{i-1} \partial_{t}^{j} \phi\right), \\
\Im\left(\tau, R^{i} \partial_{t}^{j} \phi\right) \leq C_{\tau} \Im\left(\tau+4, R^{i} \partial_{t}^{j-1} \phi\right) .
\end{array}\right.
$$

Now, if we successively apply these last inequalities for $1 \leq i \leq k_{1}$ and $1 \leq$ $j \leq k_{2}$ we deduce the existence of two positive constants $\widetilde{C}_{\tau}$ and $\widetilde{\sigma}_{\tau}$ (depending on $\Omega, \omega, n,\left(r_{i j}\right)_{1 \leq i, j \leq N}, D, A, \tau, k_{1}$ and $\left.k_{2}\right)$ for which

$$
\Im\left(\tau, R^{i} \partial_{t}^{j} \phi\right) \leq \widetilde{C}_{\tau} \Im(\tau+4(i+j), \phi)
$$

holds for every $s \geq \widetilde{\sigma}_{\tau}\left(T+T^{2}+T^{2}\|c\|_{\infty}^{2 / 3}\right)$ and for every $i, j$ with $0 \leq i \leq k_{1}$ and $0 \leq j \leq k_{2}$. Finally, choosing $\tau=\tau_{0}-4(i+j)$ and taking into account inequality (3.22), we obtain (3.17).

Remark 3.2. As far as the Carleman estimate is concerned, it is enough to assume that the diffusion coefficients satisfy $d_{i} \neq 0$ for $1 \leq i \leq n$.

\subsection{Proof of Theorem 1.2. The Carleman inequality for the adjoint problem}

We will devote this section to show the Carleman estimate for the adjoint problem (1.8) stated in Theorem 1.2. Let us recall that $\mathcal{D}$ stands for the space $\mathcal{D}=\bigcap_{p=0}^{\infty} D\left(R^{p}\right)$, a space which is dense in $L^{2}(\Omega)$.

The starting point relies on the following observation.

Proposition 3.3. Let us consider $\varphi_{0} \in \mathcal{D}^{n}$ and let $\varphi=\left(\varphi_{1}, \cdots, \varphi_{n}\right)^{*}$ be the corresponding solution of problem (1.8). Then, $\varphi \in C^{k}\left([0, T] ; D\left(R^{p}\right)^{n}\right)$ for every $k, p \geq 0$, and, for every $i$ (with $1 \leq i \leq n$ ) $\varphi_{i}$ solves (3.16).

Proof. Assume $\varphi_{0} \in \mathcal{D}^{n}$ and let $\varphi=\left(\varphi_{1}, \cdots, \varphi_{n}\right)^{*}$ be the corresponding solution of problem (1.8). Setting $P^{*} \psi(x, t)=\varphi(x, T-t)$ for $(x, t) \in \Omega_{T}, \psi$ solves problem:

$$
\left\{\begin{array}{l}
\partial_{t} \psi=\left(J R+\left(P^{*}\right)^{-1} A^{*} P^{*}\right) \psi \text { in } \Omega_{T} \\
\psi=0 \text { on } \Sigma_{T}, \quad \psi(x, 0)=\left(P^{*}\right)^{-1} \varphi_{0} \text { in } \Omega
\end{array}\right.
$$

(see (1.5) for the definition of $J$ and $P$ ). The operator $J R$ with domain $D(J R)=$ $\left(H^{2}(\Omega) \cap H_{0}^{1}(\Omega)\right)^{n}$, is dissipative and self adjoint on the space $L^{2}(\Omega)^{n}$ : it is then 
the generator of an analytic semigroup. Since $\left(P^{*}\right)^{-1} A^{*} P^{*}$ is a bounded linear operator on $L^{2}(\Omega)^{n}$, by the perturbation theory of analytic semigroups (see [21, Corollary 2.2., p. 81]), the operator $J R+\left(P^{*}\right)^{-1} A^{*} P^{*}$ is also the generator of an analytic semigroup. Since $D\left(\left(J R+\left(P^{*}\right)^{-1} A^{*} P^{*}\right)^{p}\right)=D\left(R^{p}\right)^{n}$, the regularity part follows from the semigroup theory.

Let $Q(\eta, \xi)=\eta I+D^{*} \xi+A^{*}$ for $(\eta, \xi) \in \mathbb{R}^{2}$ and set $Q\left(\partial_{t}, \nabla\right)=\partial_{t}+$ $D^{*} R+A^{*}:=\left(Q_{i j}\left(\partial_{t}, \nabla\right)\right)_{1 \leq i, j \leq n}$. As operators acting on $C^{\infty}\left([0, T] ; \mathcal{D}^{n}\right)$, all the entries $Q_{i j}\left(\partial_{t}, \nabla\right)$ live this space invariant and commute between them. So, as it is done in [15, I.VI.4, p. 144], in the system $Q\left(\partial_{t}, \nabla\right) \varphi=0$, we carry out the (Gauss) algebraic elimination process as if the operators were constants. With $P\left(\partial_{t}, \nabla\right)=\operatorname{det} Q\left(\partial_{t}, \nabla\right)$, the conclusion follows.

Proof of Theorem 1.2: Firstly, we will assume that $\varphi_{0} \in \mathcal{D}^{n}$. Let $\varphi$ be the solution to (1.8) corresponding to $\varphi_{0}$. We can then apply Proposition 3.3 and deduce that $\varphi \in C^{\ell}\left([0, T] ; D\left(R^{p}\right)^{n}\right)$, for every $\ell, p \geq 0$, and $\varphi_{i}$ satisfies (3.16) for every $i, 1 \leq i \leq n$. Also, $\left(B^{*} \varphi\right)_{j}$ lies in $C^{\ell}\left([0, T] ; D\left(R^{p}\right)^{n}\right)$ and solves $(3.16)$, for all $j$ (with $1 \leq j \leq m$ ).

Thus Theorem 3.2 can be applied to $\phi=\left(B^{*} \varphi\right)_{i}(1 \leq i \leq m)$ with $k_{1}=k$, $k_{2}=n-1$ and $\tau_{0}=\tau-4 k-n+4$ deducing the existence of two positive constants $\widetilde{C}$ and $\widetilde{\sigma}$ (only depending on $\Omega, \omega, n,\left(r_{i j}\right)_{1 \leq i, j \leq N}, D, A, k$ and $\tau$ ) such that

$$
\sum_{l=0}^{k} \sum_{j=0}^{n-1} \Im\left(\tau_{0}-4(l+j), R^{l} \partial_{t}^{j}\left(B^{*} \varphi\right)_{i}\right) \leq \widetilde{C} \iint_{\omega_{T}}(s \rho)^{\tau_{0}+r} e^{-2 s \eta}\left|B^{*} \varphi\right|^{2}
$$

for every $s \geq \widetilde{s}:=\widetilde{\sigma}\left(T+T^{2}+T^{2}\|c\|_{\infty}^{2 / 3}\right)$. Observe that thanks to (3.24), if $s \geq \widetilde{s}$ we can affirm

$$
(s \rho(t))^{\tau} \leq C(s \rho(t))^{\tau_{0}-4(l+j)+3 n}, \quad \forall t \in(0, T),
$$

for every $l, j$ with $0 \leq l \leq k, 0 \leq j \leq n-1$. The definition of $\mathfrak{I}$ (see the statement of Theorem 3.2) and $K$ leads immediately to

$$
\sum_{j=0}^{n-1} \iint_{\Omega_{T}}(s \rho)^{\tau} e^{-2 s \eta}\left|R^{k} \partial_{t}^{j}\left(B^{*} \varphi\right)_{i}\right|^{2} \leq \widetilde{C} \iint_{\omega_{T}}(s \rho)^{\tau+K+r} e^{-2 s \eta}\left|B^{*} \varphi\right|^{2}
$$

for every $s \geq \widetilde{\sigma}\left(T+T^{2}+T^{2}\|c\|_{\infty}^{2 / 3}\right)$ and $i=1, \cdots, m$. In (3.28), $r=r(n)$ is as in the statement of Theorem 3.2 and $K=4 k+n-4$.

On the other hand, using (1.8) and the expression of $\mathcal{K}^{*}$ we get:

$$
\mathcal{K}^{*} \varphi(\cdot, t) \equiv\left((-1)^{n-1} \partial_{t}^{n-1} B^{*} \varphi,(-1)^{n-2} \partial_{t}^{n-2} B^{*} \varphi, \cdots,-\partial_{t} B^{*} \varphi, B^{*} \varphi\right)^{*}(\cdot, t)
$$

in $[0, T)$. Now, if we set $M_{0}=\max _{\bar{\Omega}} \beta_{0}$ and use (3.28) we infer (1.10) for every $s \geq \widetilde{\sigma}\left(T+T^{2}+T^{2}\|c\|_{\infty}^{2 / 3}\right)$. This concludes the proof in the case $\varphi_{0} \in \mathcal{D}^{n}$.

The general case can be easily obtained from a density argument. Indeed, if $\varphi_{0} \in L^{2}(\Omega)^{n}$ there exists a Cauchy sequence $\left\{\varphi_{0}^{\ell}\right\}_{\ell \geq 1} \subset \mathcal{D}^{n}$ such that $\varphi_{0}^{\ell} \rightarrow \varphi_{0}$ 
in $L^{2}(\Omega)^{n}$. If $\varphi^{\ell}$ and $\varphi$ are, respectively, the solution to (1.8) corresponding to $\varphi_{0}^{\ell}$ and $\varphi_{0}$, we have $\varphi^{\ell} \rightarrow \varphi$ in $L^{2}(Q)^{n}$ and $R^{k} \mathcal{K}^{*} \varphi^{\ell} \rightarrow R^{k} \mathcal{K}^{*} \varphi$ in $\mathcal{D}^{\prime}\left(\Omega_{T}\right)^{n}$ for every $k \geq 0$. Observe that $\left(\varphi^{\ell}, \varphi_{0}^{\ell}\right)$ satisfies inequality (1.11) for every $\ell \geq 1$. Also, inequality (1.11) implies that $\left\{R^{k} \mathcal{K}^{*} \varphi^{\ell}\right\}_{\ell \geq 1}$ is a Cauchy sequence in the weighted space $L^{2}\left((s \rho)^{\tau / 2} e^{\frac{-s m_{0}}{t(T-t)}} ; \Omega_{T}\right)$. These previous considerations permit to pass to the limit in the Carleman inequality (1.11) satisfied by $\left(\varphi^{\ell}, \varphi_{0}^{\ell}\right)$ and obtain the result in the general case. This ends the proof.

We end the section with the proof of Theorem 1.3. As said above, at this point we will use the assumption (1.6).

Proof of Theorem 1.3: It is a direct consequence of the assumption on $\mathcal{K}^{*}$ : taking account $(2.12)$ and $(1.10)$, if $k \geq(2 n-1)(n-1)$ we deduce the existence of a new positive constant $C$ for which (1.11) holds for every $s \geq \widetilde{\sigma}\left(T+T^{2}+T^{2}\|c\|_{\infty}^{2 / 3}\right)$. This finalizes the proof.

\section{The Kalman condition. Proof of Theorem 1.1}

We will devote this section to the proof of Theorem 1.1.

Proof of Theorem 1.1: Let us begin by proving the necessary part of Theorem 1.1: It is well-known that the null-controllability is equivalent to the observability estimate (1.9) for any solution of the adjoint problem (1.8).

Suppose that $\operatorname{Ker}\left(\mathcal{K}^{*}\right) \neq\{0\}$. From Proposition 2.2 it follows that there exists $p_{0} \in \mathbb{N}^{*}$ such that

$$
\operatorname{rank} \mathcal{K}_{p_{0}}=\operatorname{rank}\left[\left(-\lambda_{p_{0}} D+A\right) \mid B\right]<n .
$$

Therefore, thanks to Kalman's rank condition applied to the ordinary differential system $y^{\prime}=\left(-\lambda_{p_{0}} D+A\right) y+B v$ we infer that this system is not controllable. Thus, there exists a non zero solution $z_{p_{0}}(t) \in \mathbb{R}^{n}$ to the associated adjoint system

$$
-z^{\prime}=\left(-\lambda_{p_{0}} D^{*}+A^{*}\right) z \text { in }(0, T),
$$

satisfying $B^{*} z_{p_{0}}(t)=0$ for every $t \in[0, T]$. Then, letting $\varphi_{0}=z_{p_{0}}(T) \phi_{p_{0}}$, where $\phi_{p_{0}}$ is the normalized eigenfunction associated with $\lambda_{p_{0}}$, it is easy to check that the function $\varphi(t, x)=z_{p_{0}}(t) \phi_{p_{0}}(x)$ is the solution of (1.8) corresponding to $\varphi_{0}$. It is non zero and satisfies $B^{*} \varphi(x, t)=0$ in $\Omega_{T}$. Obviously this solution does not satisfy the observability inequality (1.9), and thus (1.4) is not null controllable.

Remark 4.1. Observe that, if condition (1.6) is not satisfied, then system (1.4) is not null controllable even if $\omega=\Omega$.

We turn now to the sufficient part of Theorem 1.1. As said above, the exact controllability to the trajectories of system (1.4) is equivalent to the null controllability of this system. Therefore, it is enough to show the observability inequality (1.9) for the solutions of the adjoint problem (1.8). Following [10], we show inequality (1.9) combining the global Carleman inequality (1.11) and the energy inequality satisfied by the solutions to the adjoint problem. 
Let $\varphi \in L^{2}\left(0, T ; H_{0}^{1}(\Omega)^{n}\right)$ be the solution to (1.8) corresponding to $\varphi_{0} \in$ $L^{2}(\Omega)^{n}$. Thanks to the hypothesis (1.6), we can apply Theorem 1.3 with $\tau=0$ and $k=(n-1)(2 n-1)$ obtaining

$$
\int_{T / 4}^{3 T / 4} \int_{\Omega} e^{\frac{-2 s M_{0}}{t(T-t)}}|\varphi|^{2} \leq C \iint_{\omega_{T}}(s \rho)^{l} e^{-2 s \eta}\left|B^{*} \varphi\right|^{2},
$$

for every $s \geq \sigma\left(T+T^{2}+T^{2}\|c\|_{\infty}^{2 / 3}\right)$, with $l=4(n-1)(2 n-1)+n+r$ and $r$ as in Theorem 1.2. It can be easily verified that

$$
e^{\frac{-2 s M_{0}}{t(T-t)}} \geq \exp \left(-2^{5} s M_{0} /\left(3 T^{2}\right)\right), \quad \forall t \in(T / 4,3 T / 4)
$$

and, if we take $s \geq\left(l / 8 m_{0}\right) T^{2}$, also

$$
(s \rho)^{l} e^{-2 s \eta} \leq s^{l} 2^{2 l} T^{-2 l} \exp \left(-2^{3} m_{0} s / T^{2}\right) \leq\left(\frac{l}{2 e m_{0}}\right)^{l}, \quad \forall(x, t) \in \Omega_{T},
$$

where $m_{0}=\min _{\bar{\Omega}} \beta_{0}$. From this three last inequalities, we readily deduce

$$
\int_{T / 4}^{3 T / 4} \int_{\Omega}|\varphi|^{2} \leq C e^{C s / T^{2}} \iint_{\omega_{T}}\left|B^{*} \varphi\right|^{2}
$$

for every $s \geq \sigma_{1}\left(T+T^{2}+T^{2}\|c\|_{\infty}^{2 / 3}\right)$ and $\sigma_{1}=\max \left\{\sigma,\left(l / 8 m_{0}\right)\right\}(C$ is a positive constant depending on $\Omega, \omega, n,\left(r_{i j}\right)_{1 \leq i, j \leq N}, D$ and $A$ ).

On the other hand, taking into account the assumptions imposed to the operator $R$ and the matrix $D$ (see (1.2) and (1.5)), it is not difficult to get the following energy inequality satisfied by $\varphi$

$$
\frac{d}{d t}\left(e^{C\left(1+\|c\|_{\infty}\right) t}\|\varphi(\cdot, t)\|_{L^{2}(\Omega)^{n}}^{2}\right) \geq 0, \quad \forall t \in(0, T)
$$

with $C$ a positive constant depending on $D$ and $A$. From this last inequality we also obtain

$$
\left\{\begin{aligned}
\|\varphi(\cdot, 0)\|_{L^{2}(\Omega)^{n}}^{2} & \leq e^{C\left(1+\|c\|_{\infty}\right) T / 4}\|\varphi(\cdot, T / 4)\|_{L^{2}(\Omega)^{n}}^{2} \\
& \leq \frac{2}{T} e^{C\left(1+\|c\|_{\infty}\right) 3 T / 4} \int_{T / 4}^{3 T / 4} \int_{\Omega}|\varphi|^{2}
\end{aligned}\right.
$$

This last inequality together with (4.29) (with $s=\sigma_{1}\left(T+T^{2}+T^{2}\|c\|_{\infty}^{2 / 3}\right)$ ) imply the observability inequality for the solutions to the adjoint problem (1.8):

$$
\|\varphi(\cdot, 0)\|_{L^{2}(\Omega)^{n}}^{2} \leq e^{C\left(1+1 / T+\|c\|_{\infty}^{2 / 3}+T+T\|c\|_{\infty}\right)} \iint_{\omega_{T}}\left|B^{*} \varphi(x, t)\right|^{2},
$$

with $C>0$ a constant which depends on $\Omega, \omega, n,\left(r_{i j}\right)_{1 \leq i, j \leq N}, D$ and $A$. This finalizes the sufficient part and Theorem 1.1. 


\section{Some additional comments, results and open problems}

1. Approximate controllability. As a consequence of Theorem 1.1, we can prove that condition (1.6) also is a necessary and sufficient condition for the approximate controllability of system (1.4). In fact, one has

Theorem 5.1. Let us assume that D satisfies (1.5). Then, system (1.4) (with $R$ given by (1.2)) is approximately controllable at time $T$ if and only if the Kalman operator $\mathcal{K}$ satisfies (1.6)

Proof: The sufficient part can be proved taking into account the global Carleman inequality (1.11). Indeed, it is well known that system (1.4) is approximately controllable at time $T$ if and only if the adjoint problem (1.8) satisfies the following unique continuation property:

$$
\begin{gathered}
\text { "If } \varphi \in C^{0}\left([0, T] ; L^{2}(\Omega)^{n}\right) \text { is a solution to }(1.8) \text { and } B^{*}(t) \varphi \equiv 0 \text { in } \omega \times(0, T) \text {, } \\
\text { then } \varphi \equiv 0 \text { in } Q . "
\end{gathered}
$$

This property is a direct consequence of (1.11).

Finally, the necessary condition can be deduced arguing as in the proof of Theorem (1.1).

2. When condition (1.6) is fulfilled, it is also possible to compute the cost of the exact controllability to the trajectories of system (1.4). As a consequence of the observability inequality (4.30) and following the ideas of [10] we can prove:

Theorem 5.2. Let us assume that $R$ is given by (1.2) and $D$ satisfies (1.5). Let $y^{*} \in L^{2}\left(0, T ; H_{0}^{1}(\Omega)^{n}\right) \cap C^{0}\left([0, T] ; L^{2}(\Omega)^{n}\right)$ be a trajectory of system $(1.4)$ and let us fix $y_{0} \in L^{2}(\Omega)^{n}$. Then, if $(1.6)$ holds, there exists $v \in L^{2}\left(\Omega_{T}\right)^{m}$ such that the solution to (1.4) satisfies $y(\cdot, T)=y^{*}(\cdot, T)$ in $\Omega$. Moreover, for a positive constant $C$ (which only depends on $\Omega, \omega, n,\left(r_{i j}\right)_{1 \leq i, j \leq N}, D$ and $A$ ) one has

$$
\|v\|_{L^{2}(\Omega)^{m}}^{2} \leq \exp \left(C\left(1+1 / T+\|c\|_{\infty}^{2 / 3}+T+T\|c\|_{\infty}\right)\right)\left\|y_{0}-y^{*}(\cdot, 0)\right\|_{L^{2}(\Omega)^{n}}^{2} .
$$

3. It is possible to give a result on exact controllability to trajectories of system (1.4) when condition (1.6) is not fulfilled but there exists $\widehat{p} \in \mathbb{N}^{*}$ such that $\operatorname{rank}\left[\left(-\lambda_{\widehat{p}} D+A\right) \mid B\right]=n$. To be precise, let us consider

$$
\left\{\begin{array}{l}
\mathcal{J}=\left\{p \in \mathbb{N}^{*}: \operatorname{rank}\left[\left(-\lambda_{p} D+A\right) \mid B\right]=n\right\} \quad \text { and } \\
X=\operatorname{span}\left\{\phi_{p}: p \in \mathcal{J}\right\} \subset L^{2}(\Omega) .
\end{array}\right.
$$

Thanks to Remark 2.2 we can conclude that $\mathcal{J}$ is an infinite set whence we deduce that $X$ is an infinite-dimensional closed subspace of $L^{2}(\Omega)$. On the other hand, let us fix $y_{0} \in L^{2}(\Omega)^{n}$ and a trajectory $y^{*} \in L^{2}\left(0, T ; H_{0}^{1}(\Omega)^{n}\right) \cap$ $C^{0}\left([0, T] ; L^{2}(\Omega)^{n}\right)$ of system (1.4). Thus, one has: 
Theorem 5.3. Under the previous assumptions, if $y_{0}-y^{*}(\cdot, 0) \in X^{n}$, then there exists $v \in L^{2}\left(\Omega_{T}\right)^{m}$ such that the solution $y$ to system (1.4) satisfies

$$
y(\cdot, T)=y^{*}(\cdot, T) \quad \text { in } \quad \Omega .
$$

Proof: This result is a consequence of the following observability inequality for the solutions $\varphi$ of the adjoint problem (1.8) associated to initial data $\varphi_{0} \in X^{n}$ :

$$
\|\varphi(\cdot, 0)\|_{L^{2}(\Omega)^{n}}^{2} \leq C \iint_{\omega_{T}}\left|B^{*} \varphi(x, t)\right|^{2},
$$

where $C$ is a positive constant. So, let us prove this inequality.

Firstly, observe that thanks to the previous assumptions we can repeat the proof of Theorem 2.1 and infer the existence of a constant $C>0$ such that for every $\psi \in X^{n}$ satisfying $\mathcal{K}^{*} \psi \in D\left(R^{k}\right)^{n m}(k \geq(2 n-1)(n-1))$ one has $\psi \in D\left(R^{k-(2 n-1)(n-1)}\right)^{n}$ and

$$
\left\|R^{k-(2 n-1)(n-1)} \psi\right\|_{L^{2}(\Omega)^{n}}^{2} \leq C\left\|R^{k} \mathcal{K}^{*} \psi\right\|_{L^{2}(\Omega)^{n m}}^{2} .
$$

Secondly, we readily obtain that if $\varphi_{0} \in X^{n}$ then the solution $\varphi$ to (1.8) satisfies $\varphi(\cdot, t) \in X^{n}$ for every $t \in[0, T]$. Taking into account the two previous considerations and inequality (1.10) (with $\tau=0$ and $k=(2 n-1)(n-1)$ ), we get

$$
\iint_{\Omega_{T}} e^{\frac{-2 s M_{0}}{t(T-t)}}|\varphi|^{2} \leq C \iint_{\omega_{T}}(s \rho)^{K+r} e^{-2 s \eta}\left|B^{*} \varphi\right|^{2},
$$

for every $s \geq \sigma\left(T+T^{2}+T^{2}\|c\|_{\infty}^{2 / 3}\right)\left(M_{0}, \rho, K, r\right.$ and $\eta$ are as in the statement of Theorem 1.2).

Finally, The observability inequality (5.31) can be obtained from the previous Carleman inequality reasoning as in the proof of Theorem 1.1. This ends the proof.

4. Theorems 1.1, 1.2 and 1.3 are still valid if in (1.4) we consider Neuman boundary conditions instead of Dirichlet boundary conditions.

5. In our analysis we have strongly used the structure of the diffusion matrix $D$. It would be very interesting to extend the results of this work to the case in which $D$ is a general positive definite matrix. Observe that, in this case, even the case $B \equiv I d \in \mathcal{L}\left(\mathbb{R}^{n}\right)$ (i.e., a distributed control in each equation of the system) is still open.

6. The non autonomous problem. To our knowledge, the null controllability problem of (1.4) when the operators and the coupling matrices $A$ and $B$ are time-dependent is still open. We will address in details this question in the case $D=I d$ in a forthcoming paper (see [3]). On the other hand, the case in which $A$ and $B$ depend on $x$ seems to be much more complicated. A necessary and sufficient condition for the exact controllability to the trajectories of system (1.4) is open. 
7. Boundary controls. In view of known controllability results for a linear heat equation, it would be natural to wonder whether the controllability result for system (1.4) remains valid when one considers boundary controls exerted on a relative open subset $\gamma$ of the boundary $\partial \Omega$. Nevertheless, there exist negative results for some 1-d cascade linear coupled parabolic systems with $n=2$ which are null controllable in $(0, T)$ when we apply a distributed control $e_{1} v 1_{\omega}$ and they are not if we take $y=e_{1} v 1_{\gamma}$ on $\partial \Omega \times(0, T)$ as boundary control (cf. [8]). These counterexamples reveal the different nature of the controllability properties for a single heat equation and for coupled parabolic systems.

8. Extension to non smooth diffusion operators. Inspection of the proof of Theorem 1.1 shows that the operator $R$ has to satisfy only two conditions

- $R$ must be self-adjoint and uniformly elliptic as (1.3),

- $R$ must satisfy a Carleman estimate as in Theorem 3.1.

According to [6], [5] and [20], our result extends to operators $R$ as (1.2) with non smooth coefficients $r_{i j}$ (see [6], [5] and [20] for exact assumptions).

\section{References}

[1] F. Ammar-Khodja, A. Benabdallah and C. Dupaix, Null controllability of some reaction-diffusion systems with one control force, J. Math. Anal. Appl. 320 (2006), no. 2, 928-943.

[2] F. Ammar-Khodja, A. Benabdallah, C. Dupaix and M. GonzálezBurgos, Controllability for a class of reaction-diffusion systems: the generalized Kalman's condition, C. R. Acad. Sci. Paris, Ser. I 345 (2007), no. 10, 543-548.

[3] F. Ammar-Khodja, A. Benabdallah, C. Dupaix and M. GonzálezBurgos, A generalization of the Kalman rank condition for time-dependent coupled linear parabolic systems, in preparation.

[4] F. Ammar-Khodja, A. Benabdallah, C. Dupaix and I. Kostine, Null controllability of some systems of parabolic type by one control force, ESAIM Control Optim. Calc. Var. 11 (2005), no. 3, 426-448.

[5] A. Benabdallah, Y. Dermenjian, J. Le Rousseau, Carleman estimates for the one-dimensional heat equation with a discontinuous coefficient and applications to controllability and an inverse problem, J. Math. Anal. Appl. 336 (2007), no. 2, 865-887.

[6] A. Doubova, A. Osses, And J.-P. Puel, Exact controllability to trajectories for semilinear heat equations with discontinuous diffusion coefficients, ESAIM Control Optim. Calc. Var. 8 (2002), 621-661. 
[7] E. Fernández-Cara, M. González-Burgos, L. De Teresa, Nullexact controllability of a semilinear cascade system of parabolic-hyperbolic equations, Commun. Pure Appl. Anal. 5 (2006), no. 3, 637-656.

[8] E. Fernández-Cara, M. González-Burgos, L. de Teresa, About boundary controllability of cascade heat equations, In preparation.

[9] E. Fernández-Cara, L. De Teresa, Null controllability of a cascade system of parabolic-hyperbolic equations, Discrete Contin. Dyn. Syst. 11 (2004), no. 2-3, 699-714.

[10] E. FERnÁNDEZ-CARA, E. ZuAZuA, The cost of approximate controllability for heat equations: the linear case, Adv. Differential Equations 5 (2000), no. $4-6,465-514$.

[11] A. Fursikov, O. Yu. Imanuvilov, Controllability of Evolution Equations, Lecture Notes Series 34, Seoul National University, Research Institute of Mathematics, Global Analysis Research Center, Seoul, 1996.

[12] M. GonzÁlez-Burgos, R. PÉrez-García, Controllability results for some nonlinear coupled parabolic systems by one control force, Asymptot. Anal. 46 (2006), no. 2, 123-162.

[13] M. GonzÁlez-Burgos, L. DE Teresa, Controllability results for cascade systems of $m$ coupled parabolic PDEs by one control force, submitted to Systems \& Control Letters.

[14] S. Guerrero Null controllability of some systems of two parabolic equations with one control force, SIAM J. Control Optim. 46, (2007), no. 2, 379-394.

[15] E. L. IncE. Ordinary Differential Equations, Dover Publications, New York, 1944.

[16] O. Yu. Imanuvilov, M. Yamamoto, Carleman inequalities for parabolic equations in Sobolev spaces of negative order and exact controllability for semilinear parabolic equations, Publ. Res. Inst. Math. Sci. 39 (2003), no. 2, $227-274$.

[17] R. E. Kalman, P. L. Falb and M. A. Arbib, Topics in Mathematical Control Theory, McGraw-Hill Book Co., New York-Toronto, Ont.-London 1969.

[18] G. Lebeau, L. Robbiano Contrôle exact de l'équation de la chaleur, Comm. Partial Differential Equations 20 (1995), no. 1-2, 335-356.

[19] H. LEIVA, Controllability of a system of parabolic equations with nondiagonal diffusion matrix, IMA J. Math. Control Inform. 22 (2005), no. 2, 187-199. 
[20] J. Le Rousseau, Carleman estimates and controllability results for the one-dimensional heat equation with BV coefficients, J. Differential Equations 233 (2007), no. 2, 417-447.

[21] A. PAZY, Semigroups of linear operators and application to partial differential equations, Applied Mathematical Sciences, 44, Springer-Verlag, New York, 1983.

[22] L. DE TERESA, Insensitizing controls for a semilinear heat equation, Comm. Partial Differential Equations 25 (2000), no. 1-2, 39-72.

[23] R. TRIgGiani, Constructive steering control functions for linear systems and abstract rank conditions, J. Optim. Theory Appl. 74 (1992), no. 2, $347-367$.

[24] J. ZABCZYK, Mathematical Control Theory: An Introduction, Systems \& Control: Foundations \& Applications, Birkhäuser, Boston, 1992. 